\title{
Nonhuman Primate Models of Stroke for Translational Neuroprotection Research
}

\author{
Douglas J. Cook • Michael Tymianski
}

Published online: 22 March 2012

(C) The American Society for Experimental NeuroTherapeutics, Inc. 2012

\begin{abstract}
Despite the discovery of several promising neuroprotective therapies in rodent models of stroke, no therapy other than the fibrinolytics has been found to be effective in human clinical trials. To address potential discrepancies between rodent and human studies, the Stroke Therapy Academic Industry Roundtable (STAIR) committee suggested that nonhuman primates (NHPs) be used for preclinical, translational stroke studies. Due to the paucity of stroke studies in NHPs, few experimental models have been described. Critical factors in designing NHP stroke models include the choice of species, the method of inducing the stroke and the choice of outcome measures. In this review, we describe established NHP models of stroke and discuss factors that may influence model development with a focus on models that may be useful in preclinical studies for neuroprotective drug screening prior to clinical trials.
\end{abstract}

Keywords Stroke $\cdot$ Nonhuman primate $\cdot$ Macaque $\cdot$ Models of disease $\cdot$ Clinical trials $\cdot$ Translational research

\section{Introduction}

Stroke is a leading cause of death and disability worldwide $[1,2]$. Thrombolytic therapy is currently the only widely used treatment in the acute phases of stroke and works by restoring blood flow to the ischemic brain before permanent damage

\section{J. Cook $\cdot$ M. Tymianski $(\bowtie)$}

Division of Neurosurgery, Department of Surgery,

University of Toronto, Toronto Western Hospital

Division of Neurosurgery, University Health Network,

Toronto Western Research Institute, University Health Network,

4-435 West Wing, 399 Bathurst St.,

Toronto, Ontario, Canada M5T 2S8

e-mail: Mike.Tymianski@uhn.on.ca ensues. However, thrombolytic therapies are only used in a small proportion of stroke patients due to a limited therapeutic window, contraindications to therapy, and limited access in many regions [3, 4]. An alternative to reperfusion therapy for acute ischemic stroke is "neuroprotection," the strategy that increases the resiliency of the brain to acute ischemia $[5,6]$. However, the translation of neuroprotective stroke therapies from animal studies to clinical trials has been challenging as all neuroprotective compounds deemed to be effective in rodent models of stroke have been ineffective in humans $[7,8]$. These challenges gave rise to concerns that neuroprotection may not be a feasible or practicable strategy in humans. Although the translational barriers between rodents and humans are poorly defined, the anatomical and physiological differences between the rodent and human brain have been frequently cited as potential reasons [9-12].

The Stroke Therapy Academic Industry Roundtable (STAIR) was a collaborative meeting held for the first time in 1999 among stakeholders in industry and academia. It was held to discuss potential reasons for past failures in translating promising therapeutics to human usefulness, and to make recommendations for future directions in neuroprotection research [13]. The committee's recommendations suggested that after sufficient evidence of efficacy was obtained for a given therapy in rodent models, "stroke recovery studies in primates might be done with a gyrencephalic species, similar to humans (e.g., macaque monkeys) [13]. This recommendation was reinforced at the Sixth STAIR meeting in 2009, and by others $[3,8,9,12,14-17]$. However, it should be noted that the STAIR committee's recommendations have never been validated. Although these recommendations are aimed at improving the methodological quality of preclinical animal research, they have yet to lead to a neuroprotective treatment in humans. 
The notion that stroke studies in nonhuman primates (NHPs) will enable the translation of a promising therapy to humans is theoretically sound, but unproven in the absence of a positive human study. Moreover, tissue plasminogen activator (the only widely approved acute ischemic stroke therapy) was translated to human usefulness with rabbit models of thromboembolic stroke, and not primates $[18,19]$. Conversely, clinical trials with the neuroprotectant NXY-059 were conducted after promising preclinical results in NHPs, but they resulted in a failed phase III human trial [20-23]. Thus, factors other than the animal model may need to be accounted for in the effort to translate promising neuroprotectants to clinical usefulness.

In addition to barriers imposed by possible anatomical and physiological differences between species, the development of neuroprotectants must contend with some serious practicalities. The large number of failed human trials that followed preclinical studies conducted primarily in rodents have reduced current levels of interest among the industry to invest in neuroprotection research. Thus, a reality in the development of putative neuroprotectants is that funding such research is challenging, and evidence of promise for human trials must meet an extremely high burden of proof. Such proof may require preclinical validation in NHPs. Therefore, further work with NHP stroke models is potentially valuable to determine their usefulness in preclinical screening and in the discovery of novel stroke therapies.

\section{Ethical Challenges}

The ethical challenges inherent in primate research must be defined and carefully considered in contemplating a primate stroke model. Any use of an experimental animal in stroke research must be accompanied by an effort to eliminate or minimize pain, distress, emotional anxiety, and/or depression related to neurological deficits in the animal subjects [24]. These effects may be more evident in species such as NHPs in which the social structure and relationships are a major part of normal life and are potentially distorted or removed in the presence of disability from neurological disease. Although analgesia, environmental enrichment, and intensive veterinary care can compensate for these factors to some extent, 1 cannot judge the degree to which discomfort is relieved in the absence of a complete understanding of how stroke affects the personal and social norms for these species. The argument often made to justify primate stroke research weighs the discomfort encountered by primate subjects against risk avoidance in subsequent human studies and potential benefits to humans through new discoveries [25]. However, the lack of insight into the extent and perception of neurological deficits produced within primate subjects and colonies leads to weighing discomfort in primate subjects against benefits in humans
[26]. Hence, it is our view that pre-clinical NHP stroke studies involving recovery and follow-up after stroke, in which animals are potentially exposed to discomfort and major alterations in social structure, should only be contemplated in screening promising agents that have a strong evidence base. These therapies should include meeting requirements for good scientific inquiry, such as certain ones suggested by the STAIR criteria. Moreover, in pre-clinical NHP testing, there should be a clear commitment to carry the therapy forward to human clinical trials. The primate studies should be undertaken in a manner that limits the potential for distress and pain as much as possible, and maximizes steps to relieve these. Therefore, a team of experienced research staff should undertake such projects with frequent review to minimize complications, to recognize and treat pain and distress, and to make decisions regarding the early termination of experiments, including, when necessary, euthanasia of distressed animals.

The aforementioned argument does not contemplate the use of NHPs in nonpreclinical studies including fundamental molecular, physiological, and imaging studies. However, there are instances in which NHPs are deemed to be required for such studies, and our views do not conflict with these needs. Multiple studies in single subjects should be undertaken when possible to minimize the number of subjects required and to maximize the usefulness of each subject, if the study protocol is minimally distressful to the animals used. Finally, institutional review of such projects should be regularly undertaken to ensure these criteria are met.

\section{Planning of Stroke Models in Nonhuman Primates}

The obvious goal of a primate stroke model is to make the model match the human condition as closely as possible. Although it may be argued that human stroke, itself, is characterized by high variability, specific human stroke syndromes can certainly be replicated in a laboratory setting. Thus, 1 approach is to start with the clinical stroke syndrome and outcomes of interest in an anticipated clinical trial and model those in the animal study. Collaboration between statisticians, clinicians, and scientists involved with both human trials and primate studies may be helpful in the planning stage to ensure that the primate study focuses on variables to be considered in a human trial (i.e., time to presentation, timing of treatment, drug doses, type of stroke syndromes to be included, imaging workup anticipated, and outcomes being measured) and to incorporate these into the primate study. In designing a NHP stroke study there are 3 major questions to be addressed:

1. Which NHP species should be used?

2. What stroke syndrome should be modeled?

3. What outcome measures will be studied? 
In addition to these main questions, general principles guiding any preclinical stroke study should follow as many of the principles emphasized by STAIR $[13,14]$. These include measurement and control of all physiological variables, including body temperature, blood pressure, oxygen saturation, blood glucose, and $\mathrm{pH}$; technical consistency in creating strokes; blinded drug/placebo administration; and blinded outcome data analysis. The STAIR criteria, although never validated, are a good faith attempt to limit circumstances that could cause false positive or negative results in preclinical studies like hypothermia/hyperthermia. It is sometimes impossible or impractical to comply with all of the recommendations (e.g., avoidance of neuroprotectant anesthetics). Moreover, controlling these variables is not necessarily possible in human clinical trials, particularly in the pre-hospital stages of treatment. Nonetheless, the STAIR recommendations provide guidelines for improving the methodological quality of studies so that the increased variability observed in human trials may be minimized in the preclinical evaluation of a putative therapeutic agent.

\section{Choice of Primate Species for Stroke Model}

Ideally, knowledge gained in lower order species might be translated to a primate model. However, studies that provide direct anatomical, physiological, and pathological comparisons of rodents, NHP, and human brains are limited. Only 1 study to date has directly compared strokes of rats and of NHPs. This study compared the timing of cell death in the ischemic basal ganglia of baboons and rats following transient middle cerebral artery occlusion (MCAO). This study suggested that the peak and plateau of cell death in baboons occurred at $5 \mathrm{~h}$ following ischemia onset as compared with $20 \mathrm{~h}$ in rats. The authors suggested that cell death following stroke in NHPs occurs in a similar timeframe to that anticipated in humans, and at a more rapid rate than in rats [27]. This study emphasizes the differences in pathophysiology following stroke in rodents and baboons, however, the underlying causes of this difference remain unresolved and require further research.

Primate species vary widely in brain and vascular anatomy, physiology, and behavior. Thus, some species may be better or less suited to a given research goal. Specific considerations include the degree of anatomic resemblance to the human brain (e.g., the presence of defined cortical and subcortical structures and white matter tracts and the position and extent of cortical representation for motor and sensory functions), the vascular supply, and collateral flow in the brain region(s) being studied, the technical feasibility of parent vessel occlusion and reperfusion, the ability to image the brain with clinically relevant modalities, such as computed tomography and magnetic resonance imaging, and the ability to undertake detailed neurobehavioral analysis.
Experimental stroke models have been conducted in both lissencephalic (smooth cortex) and gyrencephalic (convoluted cortex with gyri and sulci) primate species. The lissencephalic species are from the primate families Callitrichidae, Cebidae, Aotidae, Pitheciidae, and Atelidae; whereas gyrencephalic species are generally from the Cercopithecidae family. Table 1 summarizes NHP species used in stroke research.

The most commonly used lissencephalic species are Callithrix jacchus (common marmoset) and Saimiri sciureus (squirrel monkey) [28, 29]. Lissencephalic species may offer an advantage in comparison with gyrencephalic species in studies requiring consistent, focal strokes, because motor, sensory, and visual representation is on the cortical surface allowing precise functional mapping with minimal brain exposure before ischemia. By contrast, in the gyrencephalic species, mapping of specific regions may not be as precise when motor representation lies on cortex within a sulcus [28]. Precise mapping allows investigators to create highly reproducible lesions with predictable deficits, decreasing variability in outcome measures, and decreasing animal numbers to obtain statistical power in treatment studies.

Preclinical studies in the common marmoset, a lissencephalic primate, suggested that NXY-059 had promise as a neuroprotectant. However, the human study was negative [23]. The lissencephalic brain structure of the marmoset, being more rat-like than human, has been cited by critics as a potential cause of the discrepancy between these studies. However, there are no studies to date to suggest that gross brain structure predicts responsiveness to neuroprotection. Furthermore, the lissencephalic architecture of the marmoset brain may not have been the sole factor for the failure to translate NXY-059 to the human situation. Nonetheless, anatomical and as-yet uncertain biological differences between lissencephalic and gyrencephalic species add a layer of uncertainty to the translation of neuroprotectants to human studies.

Table 1 Nonhuman Primate Species Used in Stroke Research

\begin{tabular}{|c|c|c|}
\hline Species & Common name & Brain structure \\
\hline Callithrix jacchus* & Common marmoset & Lissencephalic \\
\hline Saimiri sciureus* & Squirrel monkey & Lissencephalic \\
\hline Aotus lemurinus & Owl monkey & Lissencephalic \\
\hline Galago senegalensis & Senegal bush baby & Lissencephalic \\
\hline Papio anubis* & Baboon & Gyrencephalic \\
\hline Macaca mulatta* & Rhesus macaque & Gyrencephalic \\
\hline Macaca fascicularis* & Cynomolgus macaque & Gyrencephalic \\
\hline Macaca fuscata & Japanese monkey & Gyrencephalic \\
\hline Cercopithecus aethiops & African green monkey & Gyrencephalic \\
\hline Cercopithecus pygerithus & Vervet monkey & Gyrencephalic \\
\hline
\end{tabular}

*Most commonly used species 
Consequently, the remainder of this review will be focused on gyrencephalic species used in stroke neuroprotection research.

Gyrencephalic species are generally larger and possess brains that exhibit a cortical organization, an organization of subcortical structures, including deep gray nuclei and white matter tracts like the internal capsule, white/gray matter ratios, and vascular distributions that resemble human brains more closely than brains of lissencephalic primates (Figs. 1, 2) [12, 30]. Hence, in that regard, gyrencephalic species are favorable for preclinical experimentation. However, these large NHP species generally cost more to house and provide care, given the need for larger housing facilities, increased numbers of technical staff for animal handling, and enhanced environmental and dietary enrichment [31,32].

Historically, the most common species used to conduct stroke experiments involving a MCAO has been the Papio anubis (baboon) [33]. This model requires enucleation of the eye followed by occlusion of the middle cerebral artery (MCA) and both anterior cerebral arteries to create a reproducible region of ischemia in the frontotemporal cortex, deep gray nuclei, and white matter of the MCA distribution $[34,35]$. A permanent MCAO in this particular stroke model produces marked edema requiring prolonged intensive care of the animal and is associated with a high risk of mortality. Therefore, a transient ischemia model has been developed to facilitate recovery experiments; however, this produces stroke primarily in subcortical nuclei, sparing the cortex [36-38]. The model provides an anatomical representation of human cortical anatomy with a gyrencephalic brain, similar subcortical anatomy, and equivalent white matter tracts. Unfortunately, the baboon cerebral vasculature differs from that of humans due to a rich collateralization. This necessitates the occlusion of the MCA and bilateral A1 segments of the anterior cerebral arteries to create a stroke in the MCA distribution. Neurobehavioral assessments in this model are limited to observational scoring due to the aggressive nature of the baboon and enucleation may obscure detailed cognitive, sensory, and motor neurobehavioral tasks that require intact binocular vision [39].

Macaque monkeys (genus Macaca) have a gyrencephalic brain with similar cortical and subcortical anatomy to humans (Figs. 1, 2). Macaca fascicularis (cynomolgus macaque) and Macaca Mulatta (rhesus macaque) have been used in permanent and transient MCAO models [40-50]. The vascular anatomy of the macaque closely resembles the human with less collateralization than the baboon [51]. Among the macaques, the neurovascular anatomy of the M. fascicularis may be more similar to humans in that it may have less collateralization than M. Mulatta as demonstrated by the need in the latter to occlude the ACA along with the distal MCA to produce a cortical stroke [43]. An equivalent stroke may be achieved in $M$. Fascicularis by clipping only the first segment of the MCA [40]. Macaques are relatively docile and can be easily trained and assessed for cognitive, motor, and sensory deficits using standardized tests [40]. These factors enable the use of macaques for certain aspects of acute stroke research as they most closely resemble the neurovascular anatomy of humans while enabling functional assessments through detailed neurobehavioral tasks.

\section{Choice of Stroke Syndrome}

The method of stroke creation is another factor that must be considered in model development [12]. Focal ischemia related to MCA stroke is the most common of the acute ischemic stroke presentations in humans and has been the most commonly tested in rodent and NHP stroke models. Strokes in the MCA distribution affect motor, sensory, and cognitive function, and offer several targets for functional assessment. However, these strokes have inherent variability in anatomical localization based on variations in collateral blood flow between subject animals and increased variability in neurobehavioral outcomes based on the mixed nature of deficits in a given stroke distribution. This has led some investigators to pursue more focal strokes than those covering the entire MCA distribution to control variability. For instance, models are described in specific motor MCA territories, such as those controlling the hand region to increase the sensitivity of motor assessments [28]. Focal strokes of end-artery distributions, such as the lenticulostriate arteries have also been used to produce focal motor deficits reduce anatomical and behavioral variability [52]. Strokes in other anterior and posterior cerebrovascular distributions have not been widely pursued in preclinical studies.

The method of vessel occlusion must also be considered. An open approach to the MCA through a pterional craniotomy or transorbital route is the most commonly used technique, using either a surgical clip or suture ligation to achieve vessel occlusion [36, 40]. Although the transorbital method provides relatively consistent strokes, as evaluated by imaging and histological analysis, the technique requires enucleation of the eye and prevents behavioral testing in tests that require intact binocular vision. The pterional craniotomy approach results in a potentially painful incision site with decreased function of the temporalis muscle as it is dissected; however, in skilled hands the surgery is generally well tolerated and the animal is prepared for a full battery of behavioral tests when it recovers (Fig. 2). To balance issues associated with surgical approaches, endovascular techniques have been developed to create a temporary occlusion of the MCA by wedging a microcatheter tip into the MCA origin $[51,53]$. This method also requires an occlusion of the posterior cerebral artery to decrease collateral flow. Despite this, the endovascular technique appears to have increased variability in stroke size and a higher mortality rate when compared to open vascular occlusions [54]. Similarly, endovascular models have been developed that use autologous 
a

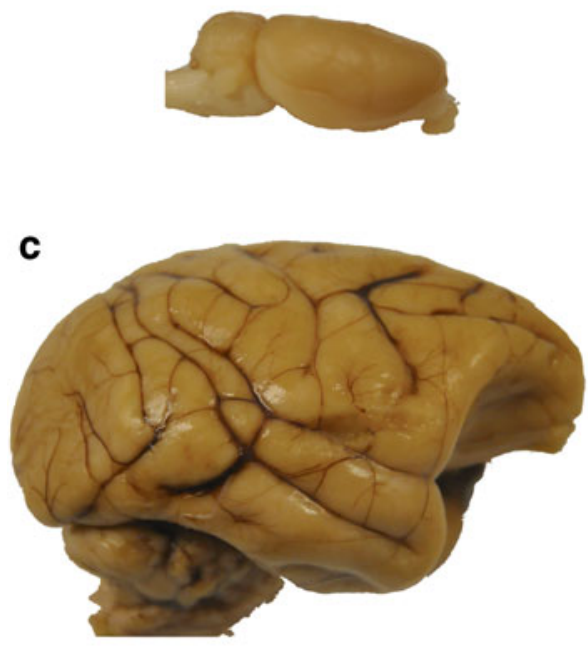

b
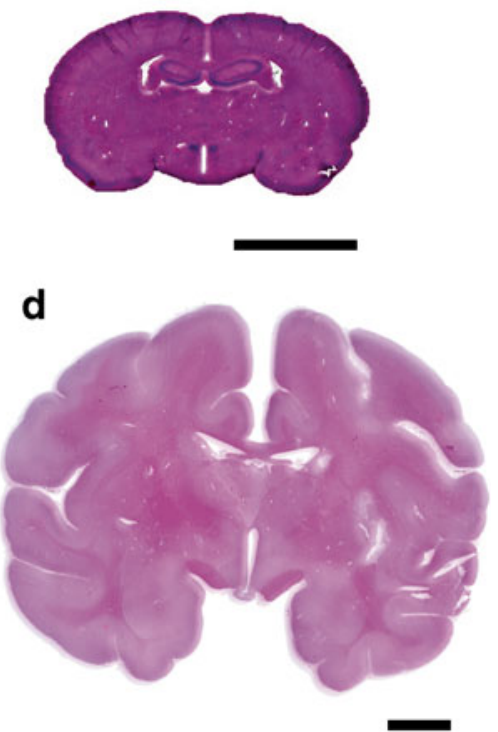

Fig. 1 Comparison of lissencephalic and gyrencephalic brain structure. (a) The rat brain is lissencephalic; gross structure demonstrates smooth cortex with absence of sulci. (b) Histological rat brain section in coronal plane stained with hematoxylin \& eosin demonstrates smooth cortical anatomy and high ratio of gray:white matter. (c) Gyrencephalic cynomolgus macaque brain; gross

blood clots to achieve vessel occlusion [47-50]. These models have the advantage of more closely modeling the pathophysiology underlying thromboembolic stroke in humans with the opportunity to achieve reperfusion with standard thrombolytic therapies; however, the variability in stroke distribution, volume, and neurological outcomes in this model may be higher. Finally, photothrombotic methods have been used in limited numbers to achieve cerebral vessel occlusion in NHPs [45, 46]. This model can be undertaken with a minimal surgical opening and can be combined with thrombolytic therapies to achieve reperfusion. However, the strokes produced are generally smaller and are located in more distal vascular distributions, and they may be variable in anatomic localization [9].

Overall, stroke models in NHPs that use endovascular approaches, including the injection of autologous blood clots to model the pathophysiology of human stroke as closely as possibly exists, but at the cost of increased variability of the outcome measures. On the other hand, open surgical models can provide lower variability and lower mortality. Thus, the balance between reduced variability and clinical relevance becomes a central determinant of the choice of primate stroke model.

\section{Choice of Outcome Measures}

Outcome measures in NHP stroke research have focused on specific aspects of neurological function, including motor, structure demonstrates multiple sulci and gyri. (d) Histological cynomolgus macaque brain section in coronal plane stained with hematoxylin \& eosin demonstrates gyri and sulci, organized white matter tracts, subcortical nuclei, and a lower ratio of gray:white matter than rats. Histology scale bars $=10 \mathrm{~mm}$

sensory, and cognitive performance after stroke [39, 40, 55]. By contrast, outcome measures in clinical trials are designed to measure disability or impairment in the activities of daily living. Measures of disability include the Modified Rankin Score, Barthel Index, and Oswestry Index [56]. Recovery in a single aspect of neurological function (e.g., motor function in the hand) may not be accompanied by an improvement in overall disability if the magnitude of recovery is small, or if there are deficits in another aspect of neurological function that prevent improvement in an activity of daily living (e.g., sensory neglect preventing patient from dressing left side). Another major difference between primate outcomes and human disability scoring is the need to train primates to perform a set behavior for a period of time, up to months, prior to the stroke; whereas, humans are assessed using existing behaviors and novel motor/cognitive tasks that introduce variation into inter-subject assessments. Therefore, there is discordance between the types of outcome measurements used in human clinical trials and ones that are achievable in primate experiments. In the absence of a disability index for use in primate studies, a battery of neurobehavioral tests can be used to gain insight into global stroke recovery in NHPs by providing data on motor, balance, sensory, perception, cognition, and basic reflexive behaviors. Specific outcome scales, such as the NHP Stroke Scale have been developed to replicate clinical scoring systems, such as the National Institutes of Health stroke scale that incorporate all of these functions [39, 40]. In the NHP Stroke Scale level of consciousness, motor function, fine 
Fig. 2 Comparison of human and nonhuman primate brain in vivo and with neuroimaging. (a) Intraoperative photo of a cynomologus macaque brain following the exposure of the Sylvian fissure. Gyri and sulci are demonstrated with cortical pial vasculature. (b)

Intraoperative photo of human brain following exposure of the Sylvian fissure demonstrating similarities to the nonhuman primate brain. (c) Axial T2weighted magnetic resonance imaging (MRI) of cynomolgus macaque at the level of the caudate nucleus demonstrating gyri and sulci, Sylvian fissure, and organized subcortical white matter tracts and nuclei. (d) Axial T2-weighted MRI of the human brain at the level of the caudate nucleus demonstrating close similarity to the organization of the macaque brain with the addition of more gyri and sulci in the cortex. (e) Retrograde brachiocephalic angiogram of cynomolgus macaque demonstrating the circle of Willis, azygous anterior cerebral artery, and middle cerebral artery "candelabra." (f) Computerized tomographic angiogram of human cerebral circulation demonstrating similar anatomy to the macaque except the presence of 2 anterior cerebral arteries and an anterior communicating artery
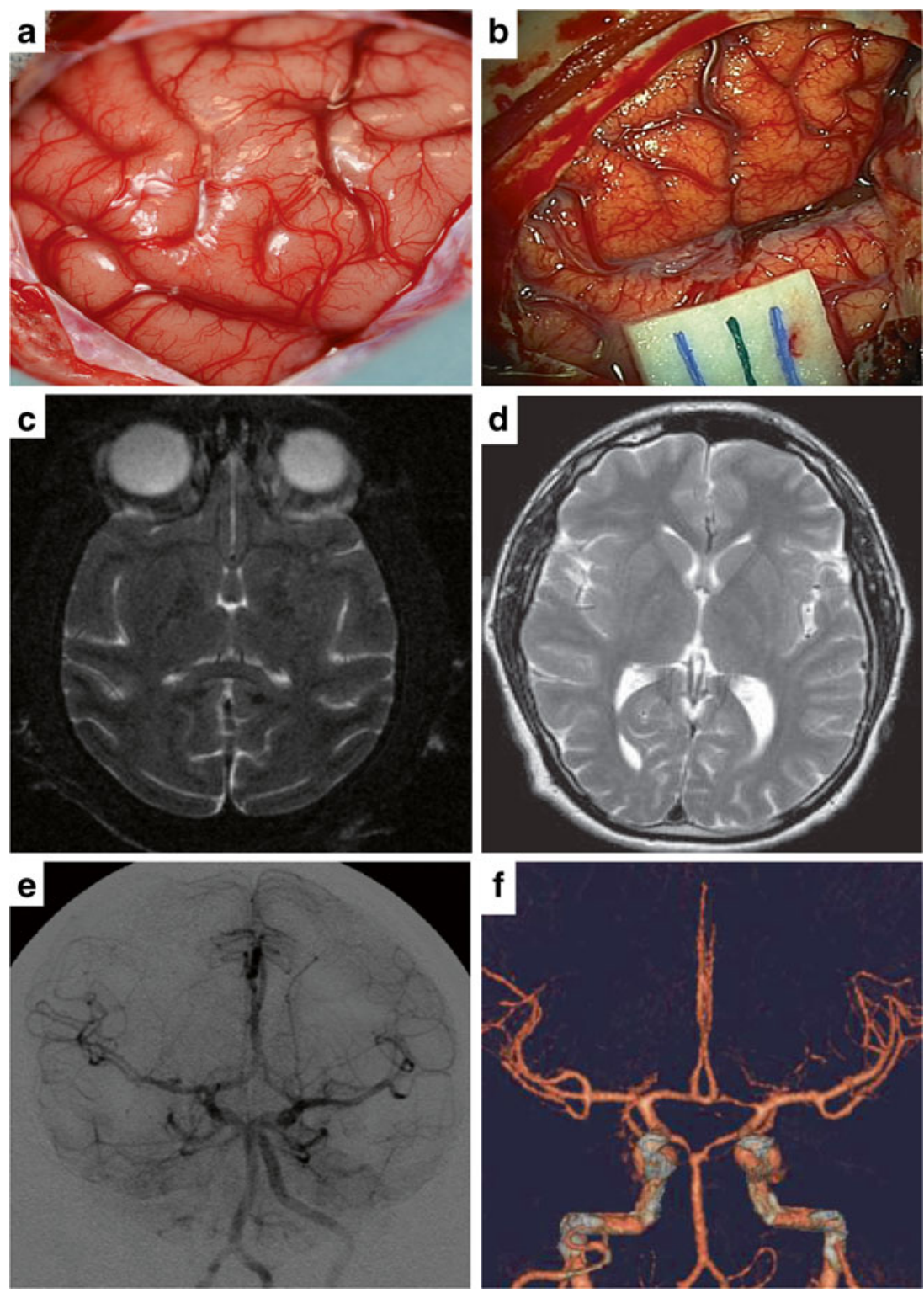

motor function, gait, balance, visual fields, feeding behaviors, defense/startle behaviors, and cranial nerves are all included in the conglomerate score.

More specific neurobehavioral tests may be used to focus on specific areas of neurological dysfunction and recovery after stroke, and can be chosen based on the anticipated deficits from the stroke created. There are several methods to assess motor function and sensory neglect in the upper extremities with tasks designed to separate the 2 . These tests are pertinent following MCA stroke. To undertake these types of tasks, animals must be trained to achieve minimum baseline scores prior to stroke onset. For example, the 2 tube choice test is a test of hand preference and hemi-spatial neglect [29, 40]. The hill and valley staircase tasks separate hemiparesis from hemi-sensory neglect by testing motor function in each arm in the isolated hemi-sensory field [29]. The Kluver board task is used to test motor control and planning [29]. Both latency (e.g., time to complete the entire task) and accuracy (e.g., deducting the score for dropped rewards) can be examined with these tasks, which provide similar information to human outcome scales, such as the Fugl-Meyer assessment of sensorimotor recovery after stroke and the Action Research Arm Test assessment of upper limb motor function [57, 58].

Cognitive tasks play a potential role in assessing impairments of memory and learning after stroke and can play a major role in determining levels of disability after a stroke [59, 60]. Cognitive function includes perception, problem solving, and memory. In the case of motor planning in complex tasks the Object Retrieval Detour Task is designed to evaluate the cognitive elements of motor planning in reaching movements and has been used in cynomolgus macaques with chronic stroke $[40,59]$. The simplest test of working memory in NHPs 
is the delayed response with or without visual discrimination [61, 62]. Higher cognitive function can be assessed with a battery of 3 tests, including the delayed nonmatching to sample task, the delayed recognition span task, and the conceptual set shifting task that have been used in studies of dementia in aged Rhesus macaques [63]. They evaluate recognition memory, pattern recognition, visuospatial memory, and executive functions [63-65]. Although these tasks have not been specifically used in stroke research, they could conceivably become incorporated in a battery of outcome measures for chronic stroke recovery in stroke models that may include cognitive deficits.

Beyond functional outcomes, surrogate markers may be added to NHP studies to acquire additional or corroborating data. As previously discussed, the validity of surrogate measures in estimating functional outcome has been questioned and remains an area of debate [66, 67]. However, in small primate studies, in which limited experience makes sample size calculations difficult, these surrogate data may be useful in detecting treatment effects in the acute phase or in detecting potential treatment effects for further study that are not evident in chronic functional outcome scores due to small sample size. Measures of tissue death, metabolic dysfunction or failure, and improvements in essential cellular homeostatic function are frequently used as surrogate measures of tissue integrity to detect neuroprotection. Tissue death is commonly measured as regions of necrotic tissue or tissue loss on histological sections; however, tissue integrity may also be measured using magnetic resonance imaging diffusion-weighted imaging and $\mathrm{T} 2$-weighted imaging as these correlate well with histology [68, 69].

Metabolic measures that correlate with tissue death have also been used as surrogate markers of stroke outcome. Of these, the vital stain 2,3,5-triphenyl tetrazolium chloride that delineates regions with functional cellular respiration from those without has close correlation to histological measures of tissue necrosis [34, 35, 70]. Positron emission tomography of metabolic activity in which thresholds of activity correlate to irreversible tissue damage have been used to measure tissue integrity in NHPs and predict resultant regions of tissue necrosis [71]. Cellular homeostatic function is disturbed after stroke with both irreversible and reversible dysfunction; however, measures of some cellular functions that correlate strongly with tissue death have the advantage of defining tissue loss based on early loss of vital intracellular processes that may precede cell death. For instance, global protein expression and gene transcription have been used in limited studies to measure cellular function after stroke [72]. Expression of the cytoskeletal protein microtubule associated protein- 2 has been used as a marker to define regions of the brain that have lost essential cellular function after stroke in NHPs [73]. Thus, there exist multiple options for the use in surrogate markers to detect and/or confirm a drug treatment effect.

\section{Conclusions}

At this stage, several questions remain concerning the clinical feasibility of neuroprotection in acute stroke and regarding the usefulness of NHPs to resolve these questions. Research to date has not resolved which primate model of stroke most closely models the human condition, nor whether studies of neuroprotectants in NHPs suffice to predict the results of a human trial. However, primate studies may allow researchers to simulate the human situation as closely as possible in the preclinical setting.

Experimental models of stroke in primates pose significant challenges, including the need for specialized infrastructure, expertise, and research costs. In our view, primate studies of neuroprotectants should only be undertaken for promising therapies that have been tested in rodent species in accordance with appropriate standards of good scientific inquiry. Ideally, studies in NHPs should correspond as closely as is practicable to a contemplated human study, and conducted if there is a commitment to conduct a clinical trial. The method of stroke creation should minimize discomfort, pain, and other deleterious effects on the planned neurological assessments. Funding mechanisms of the size and duration required for researchers to take a therapy from initial development to a human trial are generally beyond the scope of current programs available through public agencies; hence, industry support of projects or publicly funded support of programs or collaborative groups charged with the goal of translating therapies will likely be required.

The choice of NHP species depends on the study goals and the outcome measures to be used. The species selected must be capable of performing the neurobehavioral tasks for a given study, including the ability to be pre-trained to achieve consistent results in tasks prior to stroke onset. Larger species are generally capable of a greater battery of tasks including complex assessments of motor planning, fine motor control, memory and learning. Finally, the need for anatomic and physiological similarities between the primate and human brain is an area of uncertainty. Gyrencephalic brains more closely resemble the human brain on a superficial level. However, the significance of this on predicting outcome is unknown.

The development and use of NHP models of stroke have the potential to bridge the translation of neuroprotective therapies from early preclinical studies in rodents to human clinical trials. Whether results from NHPs will predict the results of human trials and lead to the discovery of a novel therapy for stroke remains to be seen.

Required Author Forms Disclosure forms provided by the authors are available with the online version of this article. 


\section{References}

1. Lloyd-Jones D, Adams RJ, Brown TM, et al. Heart disease and stroke statistics-2010 update: a report from the American Heart Association. Circulation 2010;121:e46-e215.

2. Zorowitz RD, Chen E, Tong KB, Laouri M. Costs and rehabilitation use of stroke survivors: a retrospective study of Medicare beneficiaries. Top Stroke Rehabil 2009;16:309-320.

3. Gladstone DJ, Black SE, Hakim AM. Toward wisdom from failure: lessons from neuroprotective stroke trials and new therapeutic directions. Stroke 2002;33:2123-2136.

4. Barber PA, Zhang J, Demchuk AM, Hill MD, Buchan AM. Why are stroke patients excluded from TPA therapy? An analysis of patient eligibility. Neurology 2001;56:1015-1020.

5. Lo EH, Dalkara T, Moskowitz MA. Mechanisms, challenges and opportunities in stroke. Nat Rev Neurosci 2003;4:399-415.

6. Lo EH. Degeneration and repair in central nervous system disease. Nat Med 2010;16:1205-1209.

7. Kidwell CS, Liebeskind DS, Starkman S, Saver JL. Trends in acute ischemic stroke trials through the 20th century. Stroke 2001;32:1349-1359.

8. O'Collins VE, Macleod MR, Donnan GA, et al. 1,026 experimental treatments in acute stroke. Ann Neurol 2006;59:467-477.

9. Cook DJ, Tymianski M. Translating promising preclinical neuroprotective therapies to human stroke trials. Expert Rev Cardiovasc Ther 2011;9:433-449.

10. Carmichael ST. Rodent models of focal stroke: size, mechanism, and purpose. NeuroRx 2005;2:396-409.

11. Fisher M, Finklestein S. Pharmacological approaches to stroke recovery. Cerebrovasc Dis 1999;9(suppl 5):29-32.

12. Howells DW, Porritt MJ, Rewell SS, et al. Different strokes for different folks: the rich diversity of animal models of focal cerebral ischemia. J Cereb Blood Flow Metab 2010;30:1412-1431.

13. Stroke Therapy Academic Industry Roundtable (STAIR). Recommendations for standards regarding preclinical neuroprotective and restorative drug development. Stroke 1999;30:2752-2758.

14. Fisher M, Feuerstein G, Howells DW, et al. Update of the stroke therapy academic industry roundtable preclinical recommendations. Stroke 2009;40:2244-2250.

15. Savitz SI. A critical appraisal of the NXY-059 neuroprotection studies for acute stroke: a need for more rigorous testing of neuroprotective agents in animal models of stroke. Exp Neurol 2007; 205:20-25

16. Dirnagl U. Bench to bedside: the quest for quality in experimental stroke research. J Cereb Blood Flow Metab 2006;26: 1465-1478.

17. Philip M, Benatar M, Fisher M, Savitz SI. Methodological quality of animal studies of neuroprotective agents currently in phase II/III acute ischemic stroke trials. Stroke 2009;40:577-581.

18. Zivin JA, Fisher M, DeGirolami U, Hemenway CC, Stashak JA. Tissue plasminogen activator reduces neurological damage after cerebral embolism. Science 1985;230:1289-1292.

19. NINDS tSTSG. Tissue plasminogen activator for acute ischemic stroke. The National Institute of Neurological Disorders and Stroke rt-PA Stroke Study Group. N Engl J Med 1995;333:1581-1587.

20. Marshall JW, Cummings RM, Bowes LJ, Ridley RM, Green AR. Functional and histological evidence for the protective effect of NXY-059 in a primate model of stroke when given 4 hours after occlusion. Stroke 2003;34:2228-2233.

21. Marshall JW, Duffin KJ, Green AR, Ridley RM. NXY-059, a free radical-trapping agent, substantially lessens the functional disability resulting from cerebral ischemia in a primate species. Stroke 2001;32:190-198.

22. Marshall JW, Green AR, Ridley RM. Comparison of the neuroprotective effect of clomethiazole, AR-R15896AR and NXY-059 in a primate model of stroke using histological and behavioural measures. Brain Res 2003;972:119-126.

23. Shuaib A, Lees KR, Lyden P, et al. NXY-059 for the treatment of acute ischemic stroke. N Engl J Med 2007;357:562-571.

24. Rossi J. Nonhuman primate research: the wrong way to understand needs and necessity. Am J Bioeth 2009;9:21-23.

25. Sughrue ME, Mocco J, Mack WJ, et al. Bioethical considerations in translational research: primate stroke. Am J Bioeth 2009;9:3-12.

26. Wilkinson D. Trade-offs in suffering and wellbeing: the utilitarian argument for primate stroke research. Am J Bioeth 2009;9:19-21.

27. Tagaya M, Liu KF, Copeland B, et al. DNA scission after focal brain ischemia. Temporal differences in two species. Stroke 1997;28:12451254.

28. Nudo RJ, Larson D, Plautz EJ, et al. A squirrel monkey model of poststroke motor recovery. ILAR J 2003;44:161-174.

29. Marshall JW, Ridley RM. Assessment of cognitive and motor deficits in a marmoset model of stroke. ILAR J 2003;44:153-160.

30. Hainsworth AH, Markus HS. Do in vivo experimental models reflect human cerebral small vessel disease? A systematic review. J Cereb Blood Flow Metab 2008;28:1877-1891.

31. Murphy SJ, McCullough LD, Smith JM. Stroke in the female: role of biological sex and estrogen. ILAR J 2004;45:147-159.

32. Graham SM, McCullough LD, Murphy SJ. Animal models of ischemic stroke: balancing experimental aims and animal care. Comp Med 2004;54:486-496.

33. Nehls DG, Cartwright M, Spetzler RF. Experimental primate stroke model. Neurosurgery 1986;18:388-389.

34. Spetzler RF, Zabramski JM, Kaufman B, Yeung HN. Acute NMR changes during MCA occlusion: a preliminary study in primates. Stroke 1983;14:185-191.

35. Mack WJ, Komotar RJ, Mocco J, et al. Serial magnetic resonance imaging in experimental primate stroke: validation of MRI for pre-clinical cerebroprotective trials. Neurol Res 2003; 25:846-852

36. Huang J, Mocco J, Choudhri TF, et al. A modified transorbital baboon model of reperfused stroke. Stroke 2000;31:3054-3063.

37. D'Ambrosio AL, Sughrue ME, Mocco J, et al. A modified transorbital baboon model of reperfused stroke. Methods Enzymol 2004;386:60-73.

38. Del Zoppo GJ, Copeland BR, Harker LA, et al. Experimental acute thrombotic stroke in baboons. Stroke 1986;17:1254-1265.

39. Mack WJ, King RG, Hoh DJ, et al. An improved functional neurological examination for use in nonhuman primate studies of focal reperfused cerebral ischemia. Neurol Res 2003;25:280-284.

40. Roitberg B, Khan N, Tuccar E, et al. Chronic ischemic stroke model in cynomolgus monkeys: behavioral, neuroimaging and anatomical study. Neurol Res 2003 Jan;25:68-78.

41. Murphy SJ, Kirsch JR, Zhang W, et al. Can gender differences be evaluated in a rhesus macaque (Macaca mulatta) model of focal cerebral ischemia? Comp Med 2008;58:588-596.

42. Takamatsu H, Tsukada H, Kakiuchi T, et al. Detection of reperfusion injury using PET in a monkey model of cerebral ischemia. J Nucl Med 2000;41:1409-1416.

43. West GA, Golshani KJ, Doyle KP, et al. A new model of cortical stroke in the rhesus macaque. J Cereb Blood Flow Metab 2009;29:11751186.

44. Hirouchi Y, Suzuki E, Mitsuoka C, et al. Neuroimaging and histopathological evaluation of delayed neurological damage produced by artificial occlusion of the middle cerebral artery in Cynomolgus monkeys: establishment of a monkey model for delayed cerebral ischemia. Exp Toxicol Pathol 2007;59:916.

45. Furuichi Y, Katsuta K, Maeda M, et al. Neuroprotective action of tacrolimus (FK506) in focal and global cerebral ischemia in rodents: dose dependency, therapeutic time window and long-term efficacy. Brain Res 2003;965:137-145. 
46. Li HL, Yan C, Li J, et al. Seroprevalence of Toxoplasma gondii in bred cynomolgus monkeys (Macaca fascicularis) in China. J Parasitol 2010;96:807-808.

47. Yoshikawa T, Akiyoshi Y, Susumu T, et al. Ginsenoside Rb1 reduces neurodegeneration in the peri-infarct area of a thromboembolic stroke model in nonhuman primates. J Pharmacol Sci 2008;107:32-40.

48. Kito G, Nishimura A, Susumu T, et al. Experimental thromboembolic stroke in cynomolgus monkey. J Neurosci Methods 2001; 30; $105: 45-53$

49. Kuge Y, Yokota C, Tagaya M, et al. Serial changes in cerebral blood flow and flow-metabolism uncoupling in primates with acute thromboembolic stroke. J Cereb Blood Flow Metab 2001;21:202-210.

50. Qureshi AI, Suri MF, Ali Z, et al. Intraarterial reteplase and intravenous abciximab for treatment of acute ischemic stroke. A preliminary feasibility and safety study in a nonhuman primate model. Neuroradiology 2005;47:845-854.

51. de Crespigny AJ, D'Arceuil HE, Maynard KI, et al. Acute studies of a new primate model of reversible middle cerebral artery occlusion. J Stroke Cerebrovasc Dis 2005;14:80-87.

52. Yonas H, Wolfson SK, Jr., Dujovny M, Boehnke M, Cook E. Selective lenticulostriate occlusion in the primate. A highly focal cerebral ischemia model. Stroke 1981;12:567-572.

53. Hamberg LM, Hunter GJ, Maynard KI, et al. Functional CT perfusion imaging in predicting the extent of cerebral infarction from a 3-hour middle cerebral arterial occlusion in a primate stroke model. AJNR Am J Neuroradiol 2002;23:1013-1021.

54. D'Arceuil HE, Duggan M, He J, Pryor J, de Crespigny A. Middle cerebral artery occlusion in Macaca fascicularis: acute and chronic stroke evolution. J Med Primatol 2006;35:78-86.

55. Marshall JW, Ridley RM. Assessment of functional impairment following permanent middle cerebral artery occlusion in a nonhuman primate species. Neurodegeneration 1996;5:275-286.

56. Kwon S, Hartzema AG, Duncan PW, Min-Lai S. Disability measures in stroke: relationship among the Barthel Index, the functional independence measure, and the modified rankin scale. Stroke 2004;35:918-923.

57. Fugl-Meyer AR, Jaasko L, Leyman I, Olsson S, Steglind S. The poststroke hemiplegic patient. 1. a method for evaluation of physical performance. Scand J Rehabil Med 1975;7:13-31.

58. Lyle RC. A performance test for assessment of upper limb function in physical rehabilitation treatment and research. Int J Rehabil Res 1981;4:483-492.

59. Palfi S, Conde F, Riche D, et al. Fetal striatal allografts reverse cognitive deficits in a primate model of Huntington disease. Nat Med 1998;4:963-966.
60. Dorman PJ, Sandercock PA. Considerations in the design of clinical trials of neuroprotective therapy in acute stroke. Stroke 1996;27:1507-1515.

61. Fuster JM. Unit activity in prefrontal cortex during delayedresponse performance: neuronal correlates of transient memory. $\mathrm{J}$ Neurophysiol 1973;36:61-78.

62. Zola-Morgan S, Squire LR. Preserved learning in monkeys with medial temporal lesions: sparing of motor and cognitive skills. J Neurosci 1984;4:1072-1085.

63. Herndon JG, Moss MB, Rosene DL, Killiany RJ. Patterns of cognitive decline in aged rhesus monkeys. Behav Brain Res 1997;87:2534.

64. Moore TL, Killiany RJ, Herndon JG, Rosene DL, Moss MB. Impairment in abstraction and set shifting in aged rhesus monkeys. Neurobiol Aging 2003;24:125-134.

65. Moss MB, Jonak E. Cerebrovascular disease and dementia: a primate model of hypertension and cognition. Alzheimers Dement 2007;3(2 suppl):S6-S15.

66. Johnston $\mathrm{K}$. What are surrogate outcome measures and why do they fail in clinical research? Neuroepidemiology 1999;18:167173.

67. Saver JL, Johnston KC, Homer D, et al. Infarct volume as a surrogate or auxiliary outcome measure in ischemic stroke clinical trials. The RANTTAS Investigators. Stroke 1999;30:293-298.

68. Hoehn-Berlage M, Eis M, Back T, Kohno K, Yamashita K. Changes of relaxation times (T1, T2) and apparent diffusion coefficient after permanent middle cerebral artery occlusion in the rat: temporal evolution, regional extent, and comparison with histology. Magn Reson Med 1995;34:824-834.

69. Jokivarsi KT, Hiltunen Y, Tuunanen PI, Kauppinen RA, Grohn $\mathrm{OH}$. Correlating tissue outcome with quantitative multiparametric MRI of acute cerebral ischemia in rats. J Cereb Blood Flow Metab 2010;30:415-427.

70. Bederson JB, Pitts LH, Germano SM, et al. Evaluation of 2,3,5triphenyltetrazolium chloride as a stain for detection and quantification of experimental cerebral infarction in rats. Stroke 1986; 17:1304-1308.

71. Murakami Y, Takamatsu H, Noda A, et al. Pharmacokinetic animal PET study of FK506 as a potent neuroprotective agent. J Nucl Med 2004;45:1946-1949.

72. Yanagihara T. Experimental stroke in gerbils: effect on translation and transcription. Brain Res 1978;158:435-444.

73. Kharlamov A, LaVerde GC, Nemoto EM, et al. MAP2 immunostaining in thick sections for early ischemic stroke infarct volume in nonhuman primate brain. J Neurosci Methods 2009; 182:205-210. 